J. Korean Math. Soc. 40 (2003), No. 3, pp. 423 434

\title{
APPROXIMATION OF NON-HOLOMORPHIC MAPS, AND POLETSKY THEORY OF DISCS
}

\author{
JEAN-Pierre Rosay
}

\begin{abstract}
We prove an approximation result, and we get a new proof of the main result in [7]. I hope that this new proof may be a step towards a generalization of the Poletsky theory of discs to the case of almost complex manifolds.
\end{abstract}

\section{A general question (to be made more precise)}

The general question, vaguely stated, is: Is every map from the unit disc into a complex manifold, with small $\bar{\partial}$, close to a genuine holomorphic map?

In the study of Poletsky discs, and having possible generalizations in mind (see [8] and Section 7 below), the following question arises naturally.

Let $\mathcal{M}$ be a complex manifold, equipped with some metric, and let $\Omega$ be a relatively compact region in $\mathcal{M}$. For every $\epsilon>0$, does there exist $\delta>0$ such that if $u$ is a map from the unit disc $\Delta($ in $\mathbb{C})$ into $\Omega$, with $|\bar{\partial} u| \leq \delta$, then there exists a homomorphic map $h: \Delta \rightarrow \Omega$ such that (abusing notations) $|h-u|<\epsilon$ ?

For the application, it is absolutely essential that no bound be given on $\partial u$, and that no shrinking of the disc be allowed.

If we added the hypothesis $|\partial u| \leq M$, then the existence (but not an estimate!) of $\delta$, depending on $\Omega, \epsilon$ and $M$, would result immediately from a normal family argument, arguing by contradiction.

The answer to the above question is negative in general, as was shown to me by $\mathrm{L}$. Lempert. His example is natural and embarrassingly simple. Take $\mathcal{M}$ any compact Riemann surface of genus $\geq 2$, and $\Omega=\mathcal{M}$. See

Received September 30, 2002.

2000 Mathematics Subject Classification: 32U05; Secondary 32H02, 32 Q65.

Key words and phrases: holomorphic discs, complex manifold.

Partly supported by NSF. 
Section 8. However the answer is positive for $\mathcal{M}=\mathbb{P}_{n}(\mathbb{C})$, as was shown to me by J. E. Fornaess $(n=1)$ and D. Chakrabarti.

At any rate, the general question has to be re-formulated. The result presented in Section 2 is hopefully only a step towards a better result. It is good enough for the first intended application, but one should in particular try to remove the non natural hypothesis of empty triple intersections made below.

\section{A partial result (a restricted version of the question)}

In Lempert's example the difficulty for solving the approximation problem arises even locally (at the boundary of the disc). In the intended application to the theory of discs there is no such local obstruction, because locally the maps to be approximated by holomorphic ones take value in a given coordinate patch.

This leads us to consider the following approximation problem in which the local difficulty disappears and for which we are simply left with a gluing problem.

Notations. As before $\Delta$ denotes the open unit disc in $\mathbb{C}$ and $\bar{\Delta}$ will be the closed unit disc. We fix a complex manifold $\mathcal{M}$ of complex dimension $n, \Omega_{1}, \ldots, \Omega_{R}$ some open sets in $\mathcal{M}$ with $K_{1}, \ldots, K_{R}$ compact subsets of $\Omega_{1}, \ldots, \Omega_{R}$ respectively. We assume that each $\Omega_{j}$ is biholomorphic to some open set in $\mathbb{C}^{n}$. Finally we fix $U_{1}, \ldots, U_{R}$ open sets in $\mathbb{C}$ such that $\bar{\Delta} \subset \bigcup_{j=1}^{R} U_{j}$.

We assume that all triple intersections are empty i.e. $U_{j} \cap U_{k} \cap U_{\ell}=\phi$ if $j, k, \ell$ are all distinct.

Definition. A map $\varphi$ from $\Delta$ into $\mathcal{M}$ will be called restricted if for every $j \in\{1, \ldots, R\}, \varphi\left(U_{j} \cap \Delta\right) \subset K_{j}$.

Of course that notion of "restricted" is relative to the above choices, and in order that there exists any restricted map one must have $K_{j} \cap$ $K_{k} \neq \phi$ if $\left(U_{j} \cap U_{k}\right) \cap \Delta \neq \phi$, that we will therefore assume.

We equip $\mathcal{M}$ with some Riemannian metric. It allows to give meaning to $|\bar{\partial} \varphi| \leq \delta$ if $\varphi$ is a map from $\Delta$ into $\mathcal{M}$. And if $f$ and $g$ are maps from $\Delta$ into $\mathcal{M}$ we set

$$
d(f, g)=\sup _{\zeta \in \Delta} \operatorname{dist}(f(\zeta), g(\zeta))
$$


The choice of the metric is inconsequential.

Proposition 1. With the above notations: For every $\epsilon>0$, there exists $\delta>0$ such that if $u$ is a restricted map from $\Delta$ into $\mathcal{M}$ satisfying $|\bar{\partial} u| \leq \delta$, there exists a holomorphic map $h$ from $\Delta$ into $\mathcal{M}$ such that $d(u, h) \leq \epsilon$, and $u(0)=h(0)$.

\section{The Cartan "Lemma" with bounds}

A crucial ingredient will be the following "Lemma" proved in [2].

LEMMA [2]. Let $\left(V_{j}\right)_{j=1}^{N}$ be a covering of the closed unit disc by open subsets of $\mathbb{C}$. For each $(j, k) \in\{1, \ldots, N\}^{2}$ let $g_{j k}$ be a holomorphic $(n \times n)$ matrix bounded and with bounded inverse defined on $\left(V_{j} \cap V_{k}\right) \cap \Delta$, with the usual cocycle conditions: $g_{j j}=\mathbb{1}, g_{j k}=g_{k j}^{-1}, g_{j k} g_{k \ell} g_{\ell j}=$ 11. Then there exist bounded holomorphic matrices $g_{j}$ with bounded inverses defined on $V_{j} \cap \Delta(j=1, \ldots, N)$ such that $g_{j k}=g_{k}^{-1} g_{j}$ on $\left(V_{j} \cap V_{k}\right) \cap \Delta$, with bounds for the $g_{j}$ 's and their inverses depending only on the covering and on the sup norm of the $g_{j k}$ 's and of their inverses.

Having in mind possible generalizations (to the theory of discs) it may be good to point out that an older and simpler result suffices for establishing Proposition 2 below. Roughly speaking it consists in dealing with a special case of Proposition 1 where $U_{j} \cap U_{k}=\phi$ if both $j$ and $k \neq 1$, and with the major requirement that the restriction of the map $u$ (not $h$ ) to $U_{1} \cap \Delta$ is a fixed holomorphic map from $U_{1} \cap \Delta$ into $\Omega_{1} \subset \mathcal{M}$, that extends continuously to $U_{1} \cap \bar{\Delta}$.

Then, following the proofs below, one has to find holomorphic matrices $g_{j}$ for a fixed cocycle of holomorphic matrices $g_{j k}$ continuous up to the boundary. This is essentially given by Proposition 2, page 48 in [1] (but with no bounds on the $g_{j}$ 's just in terms of bounds on the $g_{j k}$ 's). It is enough to adapt slightly the proof in [1], after adding a mild requirement on the $U_{j}$ 's in order to allow the approximation of invertible holomorphic matrices given on $\bigcup_{j \geq 2}\left(U_{j} \cap U_{1}\right) \cap \Delta$ by invertible holomorphic matrices defined on $\mathbb{C}$. Some other features of the proofs also simplify. 


\section{Proof of Proposition 1 (reduction to a nonlinear Cousin problem)}

From now on $F_{j}$ will denote a fixed biholomorphism from $\Omega_{j}$ onto an open set $F_{j}\left(\Omega_{j}\right) \subset \mathbb{C}^{n}(j=1, \ldots, R)$. Shrinking the $\Omega_{j}$ 's if needed with can assume that the derivatives $F_{j}^{\prime}$ and $\left(F_{j}^{-1}\right)^{\prime}$ have operator norms bounded by some constant $A$.

A restricted map $u$ from $\Delta$ into $\mathcal{M}$ corresponds to $R$ maps $u_{1}, \ldots, u_{R}$ respectively from $U_{j} \cap \Delta$ into $F_{j}\left(K_{j}\right) \subset \mathbb{C}^{n}$ such that on $\left(U_{j} \cap U_{k}\right) \cap \Delta$, $u_{k}=F_{k} \circ F_{j}^{-1} \circ u_{j}$.

The map $F_{k} \circ F_{j}^{-1}$ is defined on a neighborhood of $F_{j}\left(K_{j} \cap K_{k}\right.$ ) (non empty if $\left.\left(U_{j} \cap U_{k}\right) \cap \Delta \neq \phi\right)$.

Given a restricted map $u$ from $\Delta$ into $\mathcal{M}$, with sufficiently small $\bar{\partial}$ we want to approximate it by a holomorphic map $h$ from $\Delta$ into $\mathcal{M}$. It amounts to finding $R$ holomorphic maps $h_{1}, \ldots, h_{R}$ respectively from $U_{j} \cap \Delta$ into $\mathbb{C}^{n}(j=1, \ldots, R)$

(a) satisfying $h_{k}=F_{k} \circ F_{j}^{-1} \circ h_{j}$ on $\left(U_{j} \cap U_{k}\right) \cap \Delta$,

(b) and such that $h_{j}-u_{j}$ is small, as desired.

We first drop requirement (a).

If $u_{j}: U_{j} \cap \Delta \rightarrow \mathbb{C}^{n}$ is such that $\left|\bar{\partial} u_{j}\right| \leq \delta$, there exists $w_{j}^{o}: U_{j} \cap \Delta \rightarrow$ $\mathbb{C}^{n}$ such that $u_{j}+w_{j}^{o}$ is holomorphic and $\left|w_{j}^{o}\right| \leq K \delta$ (for some appropriate constant $K$ ). But we do not have

$$
u_{k}+w_{k}^{o}=F_{j} \circ F_{j}^{-1} \circ\left(u_{j}+w_{j}^{o}\right) .
$$

We only have

$$
\left|\left(u_{k}+w_{k}^{o}\right)-F_{k} \circ F_{j}^{-1}\left(u_{j}+w_{j}^{o}\right)\right| \leq\left(1+A^{2}\right) K \delta=K_{1} \delta .
$$

We wish to perturb slightly the holomorphic maps $u_{j}+w_{j}^{o}$ in order to get holomorphic maps $u_{j}+w_{j}^{o}+v_{j}$ defined on $U_{j} \cap \Delta$ such that on $\left(U_{j} \cap U_{k}\right) \cap \Delta\left(u_{k}+w_{k}^{o}+v_{k}^{o}\right)=F_{k} \circ F_{j}^{-1} \circ\left(u_{j}+w_{j}^{o}+v_{j}\right)$.

Changing notations, replacing $u_{j}+w_{j}^{o}$ by $u_{j}$ it is clear that Proposition 1 follows from the following Proposition $1^{\prime}$ which is entirely about maps in $\mathbb{C}^{n}$ (the manifold $\mathcal{M}$ not longer appears).

We shall start the next section by saying which notations we keep and by introducing some other notations in order to make it possible to read Proposition $1^{\prime}$ independently of what precedes. 


\section{Proposition $1^{\prime}$ (nonlinear Cousin problem)}

As before $U_{1}, \ldots, U_{R}$ are open sets in $\mathbb{C}$ that cover $\bar{\Delta}$ and with empty triple intersections.

For $1 \leq j<k \leq R$ we shall introduce subsets of $\mathbb{C}^{n} \omega_{j k}^{\prime}$ and $\omega_{j k}$ which correspond respectively in the previous setting to $F_{j}\left(\Omega_{j} \cap \Omega_{k}\right)$ and to the image under $F_{j}$ of the intersection of given neighborhoods of $K_{j}$ and $K_{k}$. So for $1 \leq j<k \leq R$, let $\omega_{j k}$ and $\omega_{j k}^{\prime}$ be open sets in $\mathbb{C}^{n}$ with $\omega_{j k} \subset \omega_{j k}^{\prime}$.

Let $F_{j k}$ (corresponding to $F_{k} \circ F_{j}^{-1}$ earlier) be a holomorphic immersion from $\omega_{j k}^{\prime}$ into $\mathbb{C}^{n}$. Note that we define $F_{j k}$ and $\omega_{j k}$ only for $j<k$.

Proposition 1'. With the above notations: For every $\epsilon>0$ there exists $\delta>0$ such that if for every $j \in\{1, \ldots, R\}, u_{j}$ is a holomorphic map from $U_{j} \cap \Delta$ into $\mathbb{C}^{n}$ such that for $1 \leq j<k \leq R, u_{j} u_{j}\left[\left(U_{j} \cap U_{k}\right) \cap \Delta\right] \subset$ $\omega_{j k}$ and $\left|u_{k}-F_{j k} \circ u_{j}\right| \leq \delta$, then there exist holomorphic maps $v_{1}, \ldots, v_{R}$ respectively from $U_{j}$ into $\mathbb{C}^{n}$ such that

$$
\left\{\begin{array}{l}
\left|v_{j}\right| \leq \epsilon \\
u_{k}+v_{k}=F_{j k}\left(u_{j}+v_{j}\right) \quad \text { on }\left(U_{j} \cap U_{k}\right) \cap \Delta .
\end{array}\right.
$$

Moreover if $p_{1} \in U_{1} \cap \Delta$, we can impose $v_{1}\left(p_{1}\right)=0$.

For $F_{j k}$ being the identity map this would be the standard additive Cousin Problem.

\section{Proof of Proposition $1^{\prime}$}

In order to solve $u_{k}+v_{k}=F_{j k}\left(u_{j}+v_{j}\right)$, one uses linearization. For $z \in\left(U_{j} \cap U_{k}\right) \cap \Delta$ and $t \in \mathbb{C}^{n}$

$$
F_{j k}\left(u_{j}(z)+t\right)=F_{j k}\left(u_{j}(z)\right)+\left[F_{j k}^{\prime}\left(u_{j}(z)\right)\right] t+\mathcal{O}\left(|t|^{2}\right) .
$$

The linearized problem consists in solving, for $j<k$ :

$$
\left[F_{j k}^{\prime}\left(u_{j}(z)\right)\right] v_{j}(z)-v_{k}(z)=-F_{j k}\left(u_{j}(z)\right)+u_{k}(z),
$$

where the $v_{j}$ 's have to be found, and the right hand side is given. Naturally $F_{j k}^{\prime}\left(u_{j}(z)\right)$ denotes the derivative (linear tangent map) of $F_{j k}$ at the point $u_{j}(z)$.

The Cartan Lemma with bounds allows us to write $F_{j k}^{\prime}\left(u_{j}(z)\right)=$ $g_{k}^{-1}(z) g_{j}(z)(j<k)$, where each $g_{j}$ is a holomorphic matrix defined on $U_{j}$ whose norm and whose norm of the inverse are bounded by a constant 
depending only on the $U_{j}$ 's, and on the supremum of the norms of the $F_{j k}^{\prime}$ 's and of their inverses on $\omega_{j k}$. So, and this is crucial, this constant does not depend on the $u_{j}$ 's (as long as $\left.u_{j}\left[\left(U_{j} \cap U_{k}\right) \cap \Delta\right] \subset \omega_{j k}\right)$. (*) can then be rewritten:

$$
g_{j}(z) v_{j}(z)-g_{k}(z) v_{k}(z)=g_{k}(z)\left[-F_{j k}\left(u_{j}(z)\right)+u_{k}(z)\right]
$$

By the standard additive Cousin problem (with bound, adapting Theorem 1.4 .5 in [6] in a very simple setting) there exists a continuous linear operator $T$ which to each family $\alpha=\left(\alpha_{j k}\right)_{1 \leq j<k \leq R}$ of $n$-tuples of bounded holomorphic functions $\alpha_{j k} \in\left[H^{\infty}\left(\left(U_{j} \cap U_{k}\right) \cap \Delta\right)\right]^{n}$ associates a collection $T(\alpha)=T_{1}(\alpha), \ldots, T_{R}(\alpha)$ of $n$-tuples of bounded holomorphic functions $T_{j}(\alpha) \in\left[H^{\infty}\left(U_{j} \cap \Delta\right)\right]^{n}$ such that, if $j<k$, on $\left(U_{j} \cap U_{k}\right) \cap \Delta$ : $\alpha_{j k}=T_{j}(\alpha)-T_{k}(\alpha)$. For $\alpha$ as above let $\beta=\left(\beta_{j k}\right)_{j<k}$ be defined by $\beta_{j k}=g_{k} \alpha_{j k}$ and set

$$
S_{j}(\alpha)=g_{j}^{-1} T_{j}(\beta) .
$$

Then, for $j<k, g_{j} S_{j}(\alpha)-g_{k} S_{k}(\alpha)=g_{k} \alpha_{j k}$, therefore $(* * *)$

$$
\left[F_{j k}^{\prime}\left(u_{j}(z)\right)\right] S_{j}(\alpha)-S_{k}(\alpha)=\alpha_{j k} .
$$

For $u_{1}, \ldots, u_{R}$ given let $\Phi$ be the map from $\mathcal{H}=\bigoplus_{j<k}\left(H^{\infty}\left[\left(U_{j} \cap U_{k}\right) \cap \Delta\right]\right)^{n}$ into itself defined by

$$
(\Phi(\alpha))_{j k}=\alpha_{j k}-\left[F_{j k}\left(u_{j}+S_{j}(\alpha)\right)-u_{k}-S_{k}(\alpha)\right] .
$$

We claim that given $\epsilon>0$, if all the $\left|F_{j k}\left(u_{j}\right)-u_{k}\right|$ are small enough (less than some $\delta$ ) $\Phi$ has a fixed point $\alpha$ with $|S(\alpha)| \leq \epsilon$. The corresponding family

$$
\left(v_{1}, \ldots, v_{R}\right)=\left(S_{1}(\alpha), \ldots, S_{R}(\alpha)\right)
$$

solves therefore $F_{j k}\left(u_{j}+v_{j}\right)=u_{k}+v_{k}$, with bounds as desired.

To end the proof, we have to prove the claim and it is done by a standard fixed point argument (for contractions). On each $\left(H^{\infty}\left[\left(U_{j} \cap\right.\right.\right.$ $\left.\left.\left.U_{k}\right) \cap \Delta\right]\right)^{n}(j<k)$ we consider the norm

$$
\|f\|_{j k}=\max _{m} \sup \left|f_{m}\right|, \quad f=\left(f_{1}, \ldots, f_{n}\right) .
$$

On $\mathcal{H}=\bigoplus_{j<k}\left(H^{\infty}\left[\left(U_{j} \cap U_{k}\right) \cap \Delta\right]\right)^{n}$, we can consider the norm defined by: if $\alpha=\left(\alpha_{j k}\right),\|\alpha\|=\max \left\|\alpha_{j k}\right\|_{j k}$. The closed ball of radius $\rho$ in $\mathcal{H}$ will be denoted by $B_{\rho}$.

$(* * *)$ says that $\Phi^{\prime}(0)=0$.

On a small ball around 0 of radius independent of the $u_{j}$ 's (as long as $\left.u_{j}\left[\left(U_{j} \cap U_{k}\right) \cap \Delta\right] \subset \omega_{j k}\right)$, the $\mathcal{C}^{2}$ norm of $\Phi$ is bounded independently of 
the $u_{j}$ 's (no differentiation of the $u_{j}$ 's is involved). So if $\rho>0$ is small enough, not depending on the $u_{j}$ 's $\|\Phi(\alpha)-\Phi(\beta)\|<\frac{\|\alpha-\beta\|}{2}$ for every $\alpha$ and $\beta \in B_{\rho}$. Choose furthermore $\rho \leq \frac{\epsilon}{\|S\|}(\|S\|$ the operator norm of $S$ ), we have $|S(\alpha)| \leq \epsilon$ for every $\alpha \in B_{\rho}$.

Having thus chosen $\rho$ (not depending on $u_{1}, \ldots, u_{r}$ ) we finally restrict the choice of the $u_{j}$ 's in order to have $\Phi\left(B_{\rho}\right) \subset B_{\rho}$. Since $\Phi(0)=$ $\left(-F_{j k} u_{j}+u_{k}\right)_{j k}$ and $\|\Phi(\alpha)-\Phi(0)\| \leq \frac{\|\alpha\|}{2} \leq \frac{\rho}{2}$, it is enough to require that for $j<k$

$$
\left\|F_{j k} u_{j}-u_{k}\right\|<\frac{\rho}{2}
$$

This concludes the proof of Proposition 1'.

\section{Application to the Poletsky theory of discs}

By disc (resp. holomorphic disc) in a complex manifold $\mathcal{M}$, we mean a smooth (resp. holomorphic) map from a neighborhood of the closed unit disc $\bar{\Delta}$ in $\mathbb{C}$, into $\mathcal{M}$. Abusively, for such a disc $\varphi$, we will write $\varphi: \bar{\Delta} \rightarrow \mathcal{M}$.

THEOREM. Let $f$ be an upper semi-continuous function on a complex manifold $\mathcal{M}$. For $p \in \mathcal{M}$ let

$$
\widehat{f}(p)=\operatorname{Inf} \int_{0}^{2 \pi} f \circ \varphi\left(e^{i \theta}\right) \frac{d \theta}{2 \pi},
$$

with the infimum taken over the family of all holomorphic discs $\varphi$ with $\varphi(0)=p$.

Then the function $\widehat{f}$ is plurisubharmonic.

This theorem, proved in [8], is a generalization of the ground-breaking work of Poletsky [7] (the case $\mathcal{M}=\mathbb{C}^{n}$ ), and of previous work of Lárusson and Sigurdsson [3]. See also [4] and [5].

We wish to give a new proof of the theorem. Although the proof still uses a fair amount of complex analysis (especially the existence of Stein neighborhoods for embedded discs), it avoids the ingredient which was new in [8], and which was the unpleasant construction of some Stein neighborhoods of some kind of incomplete Hartogs figure. It is our hope that part of this new proof may constitute a step towards a generalization to the case of almost complex manifolds. Although (assuming an approximation result such as Proposition 1) it has been sketched in [8], it seems more convenient to write here a complete proof, with the only 
exception that we will not repeat the proof that the function $f$ is upper semi-continuous ([3], or [8] Lemma 1).

By using approximation from above by continuous functions, it is enough to prove the theorem for (real valued) functions $f$ that are continuous, and bounded from below.

Take $p \in \mathcal{M}$ and $\varphi$ a holomorphic disc with center at $p(\varphi(0)=p)$. We have to show that

$$
\widehat{f}(\varphi(0)) \leq \int_{0}^{2 \pi} \widehat{f}\left(\varphi\left(e^{i \theta}\right)\right) \frac{d \theta}{2 \pi} .
$$

We can restrict our attention to "small" embedded discs, lying entirely in a coordinate patch.

Since the function $\widehat{f}$ is upper semi-continuous, using approximation from above (as already done in [7] (step 1)), it is enough to show that for any continuous function $v$, on $\mathbb{R} / 2 \pi \mathbb{Z}$, satisfying $v(\theta)>\widehat{f} \circ \varphi\left(e^{i \theta}\right)$

$$
\widehat{f}(\varphi(0)) \leq \int_{0}^{2 \pi} v(\theta) \frac{d \theta}{2 \pi}
$$

By definition of $\widehat{f}$ at the point $\varphi\left(e^{i \theta}\right)$, and since $v>f \circ \varphi$, for each $\theta \in[0,2 \pi]$, there exists a holomorphic disc $\psi_{0}: \bar{\Delta} \rightarrow \mathcal{M}$, such that $\psi_{\theta}(0)=\varphi\left(e^{i \theta}\right)$ and

$$
v(\theta)>\int_{0}^{2 \pi} f\left(\psi_{\theta}\left(e^{i \nu}\right)\right) \frac{d \nu}{2 \pi} .
$$

In order to deal only with embedded discs, we add one dimension. We denote by $\Pi$ the canonical projection from $\mathcal{M} \times \mathbb{C}$ onto $\mathcal{M}$. We set $\widetilde{f}=f \circ \Pi$, and let $\widetilde{\varphi}$ and $\widetilde{\psi}_{\theta}$ be the maps from a neighborhood of $\bar{\Delta}$ into $\mathcal{M} \times \mathbb{C}$, defined by:

$$
\begin{aligned}
\widetilde{\varphi}(\zeta) & =(\varphi(\zeta), 0), \\
\widetilde{\psi}_{\theta}(z) & =\left(\psi_{\theta}(z), z\right) .
\end{aligned}
$$

Note that $\tilde{\varphi}\left(e^{i \theta}\right)=\widetilde{\psi}_{\theta}(0)$. Since $\widetilde{\psi}_{\theta}$ is an embedding, there is a neighborhood $\Omega_{\theta}$ of $\widetilde{\psi}_{\theta}(\bar{\Delta})$ in $\mathcal{M} \times \mathbb{C}$, and a biholomorphism $\chi_{\theta}$ from that neighborhood onto a bounded open set $\chi_{\theta}\left(\Omega_{\theta}\right) \subset \mathbb{C}^{n+1}(n=\operatorname{dim} \mathcal{M})$. See [9], [3], or [8] Lemma 0 .

For $\theta^{\prime} \cong \theta$, instead of using the disc $\widetilde{\psi}_{\theta^{\prime}}$, we can use the disc $\widetilde{\psi}_{\theta^{\prime}}^{\theta}$ obtained from $\widetilde{\psi}_{\theta}$ by appropriate translation in the coordinates provided by $\chi_{\theta}$. Precisely, set

$$
\widetilde{\psi}_{\theta^{\prime}}^{\theta}(z)=\chi_{\theta}^{-1}\left(\chi_{\theta} \circ \widetilde{\psi}_{\theta}(z)+\chi_{\theta} \circ \widetilde{\varphi}\left(e^{i \theta^{\prime}}\right)-\chi_{\theta} \circ \widetilde{\varphi}\left(e^{i \theta}\right)\right) .
$$


For $\theta^{\prime} \cong \theta$ one still has

$$
v(\theta)>\int_{0}^{2 \pi} \widetilde{f}\left(\widetilde{\psi}_{\theta^{\prime}}^{\theta}\left(e^{i \nu}\right)\right) \frac{d \nu}{2 \pi} .
$$

For fixed $\theta,\left(\theta^{\prime}, z\right) \mapsto \widetilde{\psi}_{\theta^{\prime}}^{\theta}(z)$ is holomorphic in $z(|z|<a$, with $|a|>1)$ and smooth in $\theta^{\prime}\left(\theta^{\prime} \cong \theta\right)$.

Fix $\tau>0$. By compactness one can find disjoint intervals $J_{j} \subset \mathbb{R} / 2 \pi \mathbb{Z}$ $(j=1, \ldots, k)$, and $\theta_{j} \in J_{j}$ such that:

$$
\int_{[0,2 \pi]-\cup J_{j}}\left(|v(\theta)|+\left|f \circ \varphi\left(e^{i \theta}\right) \frac{d \theta}{2 \pi}\right|\right)<\tau,
$$

$\widetilde{\varphi}\left(J_{j}\right) \subset \Omega_{\theta_{j}}$, and for every $\theta^{\prime} \in J_{j}, \widetilde{\psi}_{\theta^{\prime}}^{\theta_{j}}(\bar{\Delta}) \subset \Omega_{\theta_{j}}$, and

$$
v\left(\theta^{\prime}\right)>\int_{0}^{2 \pi} \widetilde{f}\left(\tilde{\psi}_{\theta^{\prime}}^{\theta_{j}}\left(e^{i \nu}\right)\right) \frac{d \nu}{2 \pi} .
$$

For $d>0$ we set

$$
\begin{aligned}
& U_{2}^{j}=\left\{z \in \mathbb{C} ; \operatorname{dist}\left(z, J_{j}\right)<2 d\right\}, \\
& U_{1}=\left\{z \in \mathbb{C} ; \operatorname{dist}\left(z, \cup J_{j}\right)>d\right\} .
\end{aligned}
$$

We take $d$ small enough in order that the sets $U_{2}^{j}$ are disjoint $(j=$ $1, \ldots, k)$, and such that $\widetilde{\varphi}\left(U_{2}^{j} \cap \Delta\right) \subset \Omega_{\theta_{j}}$ (a coordinate patch).

Every smooth function, or $\mathbb{C}^{n+1}$ valued map, that is defined on some arc of the unit circle can be extended to a function with small support containing this arc, and whose $\bar{\partial}$ vanishes to infinite order along the unit circle. It can be done with holomorphic dependence on a parameter, and with bounds.

We apply it to the $\operatorname{arcs} J_{j}$ and the functions

$$
\zeta=e^{i \theta} \mapsto \chi_{\theta_{j}} \tilde{\psi}_{\theta}^{\theta_{j}}(z)
$$

where $z$ is treated as a holomorphic parameter. Then, we pull back by $\chi_{\theta_{j}}^{-1}$. Although here the notations are rather unpleasant, the goal reached is clear. Taking advantage of the fact that locally (in $\theta$ ) one works in coordinate charts. The families of holomorphic discs given on the $J_{j}$ 's (identified with arcs on the unit circle), can be extended to a family of holomorphic discs $z \mapsto \Phi(\zeta, z)$ parameterized by $\zeta$ varying in a neighborhood of $\bar{\Delta}$, with the following properties:

(i) The holomorphic discs $z \mapsto \Phi(\zeta, z)$ say in a fixed compact set in $\mathcal{M} \times \mathbb{C}$,

(ii) $\Phi(\zeta, 0) \equiv \tilde{\varphi}(\zeta)$, 
(iii) off an arbitrarily small neighborhood of the $J_{j}$ 's, in particular for $\zeta \in U_{1}$, the discs are simply constant $\operatorname{discs} \Phi(\zeta, z)=\tilde{\varphi}(\zeta)$,

(iv) for $\theta \in J_{j}, \Phi\left(e^{i \theta}, z\right)=\widetilde{\psi}_{\theta}^{\theta_{j}}(z)$,

(v) $\frac{\partial \Phi}{\partial \bar{\zeta}}=0$, for $|\zeta|=1$,

(vi) $\Phi\left(U_{2}^{j} \times \bar{\Delta}\right) \subset \Omega_{\theta_{j}}$,

(vii) $\int_{0}^{2 \pi} v(\theta) \frac{d \theta}{2 \pi} \geq \iint \tilde{f} \circ \Phi\left(e^{i \theta}, e^{i \nu}\right) \frac{d \theta}{2 \pi} \frac{d \nu}{2 \pi}-\tau$.

The following step is a key trick due to Poletsky (similar arguments were used by J. Globevnik and B. Stensones). Note that for every $N \in \mathbb{N}$,

$$
\iint \tilde{f} \circ \Phi\left(e^{i \theta}, e^{i \nu}\right) \frac{d \theta}{2 \pi} \frac{d \nu}{2 \pi}=\iint \tilde{f} \circ \Phi\left(e^{i \theta}, e^{i(\nu+N \theta)}\right) \frac{d \theta}{2 \pi} \frac{d \nu}{2 \pi} .
$$

There exists $\gamma$ (depending on $N$ ) such that

$$
\int \widetilde{f} \circ \Phi\left(e^{i \theta}, e^{i(\gamma+N \theta)}\right) \frac{d \theta}{2 \pi} \leq \iint \tilde{f} \circ \Phi\left(e^{i \theta}, e^{i \nu}\right) \frac{d \theta}{2 \pi} \frac{d \nu}{2 \pi} .
$$

This leads us to the consideration of the non holomorphic discs $\widetilde{\varphi}_{N}$ in $\mathcal{M} \times \mathbb{C}$ defined by

$$
\tilde{\varphi}_{N}(\zeta)=\Phi\left(\zeta, e^{i \gamma} \zeta^{N}\right)
$$

Note that $\bar{\partial} \widetilde{\varphi}_{N}$ tends to 0 as $N$ tends to $\infty$, due to the holomorphy in $z$, and to conditions (ii) (and the holomorphicity of $\widetilde{\varphi}$ ) and (v). (Note also that $\partial \varphi_{N}$ blows up!)

All triple intersections of $U_{1}$ and of the $U_{2}^{j}$ 's are empty. So Proposition 1 allows us to approximate $\widetilde{\varphi}_{N}$ by a genuine holomorphic disc $h_{N}$ in $\mathcal{M} \times \mathbb{C}$, with $h_{N}(0)=\widetilde{\varphi}(0)=(\varphi(0), 0)$.

We assumed that the given function $f$ is continuous. For $N$ large enough we will have

$$
\int \tilde{f} \circ h_{N}\left(e^{i \theta}\right) \frac{d \theta}{2 \pi} \leq \int \tilde{f} \circ \widetilde{\varphi}_{N}\left(e^{i \theta}\right) \frac{d \theta}{2 \pi}+\tau
$$

Since, by the very definition of $\widehat{f}$

$$
\widehat{f}(\varphi(0)) \leq \int f \circ\left(\Pi \circ h_{N}\right)\left(e^{i \theta}\right) \frac{d \theta}{2 \pi}=\int \tilde{f} \circ h_{N} \frac{d \theta}{2 \pi},
$$

it follows from the above inequalities that

$$
\widehat{f}(\varphi(0)) \leq \int v(\theta) \frac{d \theta}{2 \pi}+2 \tau .
$$

Since $\tau>0$ is arbitrary, this establishes the Theorem. 


\section{Lempert's example}

The answer to the general question raised in 1 is negative.

Proposition 2. Let $\mathcal{M}$ be a compact Riemann surface of genus $\geq 2$, endowed with some metric. There exist $\epsilon>0$ such that for every $\delta>0$ there exists a smooth map $\rho: \Delta \rightarrow \mathcal{M}$ such that $|\bar{\partial} \rho| \leq \delta$, but such that for every holomorphic map $\lambda: \Delta \rightarrow \mathcal{M} \operatorname{Sup}_{z \in \Delta} \operatorname{dist}(\rho(z), \lambda(z)) \geq \epsilon$.

So, uniform approximation of maps with small $\bar{\partial}$ is not possible.

Proof. Let $P$ be a covering map of $\mathcal{M}$, by the unit disk. Let $d$ denote the distance function on $\mathcal{M}$, and let $d_{0}$ denote the Poincare metric on $\Delta$. There exists $\epsilon>0$ such that if $f$ and $g$ are continuous maps from $\Delta$ into $\mathcal{M}$, and $\operatorname{Sup}_{z \in \Delta} d(f(z), g(z)) \leq \epsilon$, then $f$ and $g$ can be lifted to continuous maps $\widetilde{f}$ and $\widetilde{g}(f=P \circ \widetilde{f}, g=P \circ \widetilde{g})$, with $\operatorname{Sup}_{z \in \Delta} d_{0}(\tilde{f}(z), \widetilde{g}(z)) \leq 1$ (1 playing no special role).

Let $B$ be a function defined on a neighborhood of $\bar{\Delta}$ in $\mathbb{C}$ with the following properties:

(i) $\left|B\left(e^{i \theta}\right)\right| \equiv 1$,

(ii) $|B|<1$ on $\Delta$,

(iii) $B$ is holomorphic on a neighborhood of the unit circle,

(iv) for every $k \in \mathbb{N}$, the restriction of $z^{k} B$ to the unit circle does not extend holomorphically to the unit disk.

For $B$ one can take, near the unit circle, the square root of a Blaschke product with 2 simple zeroes, and cut off. Note that (i) and (iv) imply that for every holomorphic map $h: \Delta \rightarrow \Delta, \operatorname{Sup}_{z \in \Delta} d_{0}\left(h(z), z^{k} B(z)\right)=$ $+\infty$.

By a normal family argument, there exists $\alpha_{k}, 0<\alpha_{k}<1$, such that for every holomorphic map $h: \Delta \rightarrow \Delta, \operatorname{Sup}_{z \in \Delta} d_{0}(h(z),(1-$ $\left.\left.\alpha_{k}\right) z^{k} B(z)\right)>1$.

Take $\rho_{k}: \Delta \rightarrow \mathcal{M}$ defined by

$$
\rho_{k}(z)=P \circ\left(\left(1-\alpha_{k}\right) z^{k} B(z)\right) .
$$

Then $\bar{\partial} \rho_{k}$ tend to 0 uniformly on $\bar{\Delta}$, as $k \rightarrow \infty$ (due to condition (iii)). But, for every holomorphic map $\lambda: \Delta \rightarrow \mathcal{M}, \operatorname{Sup}_{z \in \Delta} d\left(\rho_{k}(z), \lambda(z)\right)>\epsilon$. Otherwise, lift $\rho_{k}$ to the map $\left(1-\alpha_{k}\right) z^{k} B(z)$. By our choice of $\epsilon$, we could lift $\lambda$ to a map $\tilde{\lambda}$ such that $\operatorname{Sup}_{z \in \Delta} d_{0}\left(\left(1-\alpha_{k}\right) z^{k} B(z), \widetilde{\lambda}(z)\right) \leq 1$, in contradiction with the choice of $\alpha_{k}$. Proposition 2 is proved. 
ACKNowledgement. I thank A. Dufresnoy for his help, and L. Lempert for allowing me to include his example in Section 8.

\section{References}

[1] A. Douady, Le problème des modules pour les sous espaces analytiques compact d'un space analytique donné, Ann. Inst. Fourier 16 (1966), 1-95.

[2] B. Berndtsson and J.-P. Rosay, Quasi-Isometric Vector Bundles and Bounded Factorization of Holomorphic Matrices, to appear in Ann. Inst. Fourier.

[3] F. Lárusson and R. Sigurdsson, Plurisubharmonic functions and analytic discs on manifolds, J. Reine Angew. Math. 501 (1998), 1-39.

[4] _ Plurisubharmonicity of envelopes of disc functionals on manifolds, preprint.

[5] A. Edigarian, A note on Rosay's paper, preprint.

[6] L. Hörmander, An Introduction to Complex Analysis in Several Complex Variables, Van Nostrand, Princeton, 1966.

[7] E. Poletsky, Plurisubharmonic functions as solutions of variational problems, Proc. Symp. Pure Math. 52 (1991), 163-171.

[8] J. P. Rosay, Poletsky theory of disks on holomorphic manifolds, Indiana Univ. Math. J. 52 (2003), 157-170.

[9] H. L. Royden, The Extension of Regular Holomorphic Maps, Proc. Amer. Math. Soc. 43 (1974), 306-310.

Department of Mathematics

University of Wisconsin

Madison WI 53706, USA

E-mail: jrosay@math.wisc.edu 